\title{
A review of alternative practices to antimicrobial use for disease control in the commercial feedlot - executive summary
}

\author{
CS Ribble DVM PhD ${ }^{1,2}$, T Stitt MPH\&TM DVM ${ }^{1}$, S Iwasawa BSc ${ }^{1}$, L Toews BA MLIS $^{3}$, C Stephen DVM PhD ${ }^{1,2}$
}

$\mathrm{T}$ he purpose of the present review was two fold: to give an overview of the modern feedlot industry in North America to provide context, and to search the scientific literature to identify alternative practices for antimicrobial use for disease control in that same industry. A fundamental assumption of the review was that reducing antimicrobial use would reduce the potential for antimicrobial-resistant bacteria to emerge and persist in the feedlot setting. To most effectively reduce antimicrobial use in the feedlot, one needs to find alternatives to prevent or effectively manage bacterial pneumonia in calves (often the principle reason for antimicrobial use) around the time of their arrival at the feedlot, as well as other bacterial diseases (eg, liver abscesses). Therefore, our key review question was the following: Are there management practices that do not involve the administration of antimicrobials that reduce the incidence of illness and mortality due to pneumonia, especially in high-risk feedlot calves? To answer this question, we set out to document the known risk factors for the emergence and maintenance of antimicrobial resistance (AMR) on feedlots, and the critical control points for these risk factors. We sought evidence for the effectiveness, efficiency and/or acceptability of various infection control points in a feedlot setting. We questioned whether the metaphylactic use of antimicrobials would affect the emergence of resistant bacteria in a feedlot setting, and asked what impact, if any, different drug use implementation strategies (including rotation of drugs used within a feedlot) might have on AMR. We also searched for management practices that reduce the incidence of liver abscesses in feedlot cattle, but that do not rely on in-feed or subtherapeutic antimicrobial use.

Our search strategy included references to management strategies, which included antimicrobial use or explored the development of AMR in an attempt to capture any articles that could contribute to answering our review questions. We did this because a preliminary search restricted to finding disease management strategies that excluded such references identified very few articles. We used this strategy to search the OVID Medline, CAB, Agricola, EMBASE and BIOSIS bibliographical databases for studies published from 1988 to April 2009. The strategy produced 2820 unique abstracts that were then subjected to a series of inclusion and exclusion criteria, which allowed us to classify them according to their relevance to our review questions, the disease or infection control strategy studied, and the strength of the results and conclusions. We then examined the literature cited in key selected articles to identify further articles of relevance to the review. This supplemental search identified 76 additional articles that were also subjected to the inclusion and exclusion criteria.

Research critically evaluating methods to prevent and control disease or the development of AMR on feedlots was rare. There were 387 articles that had some relevance to our topic, indicating a publication average of 19 articles per year over the past 20 years, or roughly three articles every two months. We classified 142 articles (5\% of the total 2896 abstracts) as ones that dealt more specifically with management approaches to reduce feedlot disease without antimicrobial use. Of these 142 articles, $25 \%$ dealt with risk factors for disease development at the feedlot, and $23 \%$ dealt with vaccination on arrival at the feedlot. Twenty-eight articles had disease management to the feedlot as their main subject; only nine of these 28 articles presented evidence from new data collected by the authors, and none of them provided information that might be useful for the design of effective disease management strategies for the large modern feedlot. The remaining articles researched nutritional management of disease (12\%), vaccination or preconditioning (a process involving weaning calves at least three weeks before sale, training them to eat from a feed bunk and vaccination) before arrival at the feedlot (10\%), and miscellaneous issues $(10 \%)$.

The 36 articles that dealt with risk factors for disease were predominantly observational studies. The majority (56\%) of these researched specific pathogens, while some researched general risk factors $(40 \%)$; a few examined animal behaviour $(8 \%)$, mixing $(6 \%)$ or transportation. A number of these observational studies were useful for understanding the epidemiology of bacterial diseases in the feedlot and developing hypotheses about alternative disease management strategies. For example, it appeared that disease at the feedlot can cluster at the levels of cow-calf sources, feedlots and pens within feedlots, although this clustering is poorly understood. Time of year animals were purchased, weight of calves purchased (a proxy for age), animal source, distance trucked (in some cases), mixing of calves from different sources, and climatic factors (specifically, total precipitation and temperature variability) were variables shown to be potentially important reasons disease clustered at the feedlot. Feedlot managers now use these

This is the executive summary from a comprehensive review with the same title. To access the full review, please visit http://nccid.calen/amr-reviews ${ }^{1}$ Centre for Coastal Health, Nanaimo, British Columbia; ${ }^{2}$ Department of Ecosystem and Public Health, Faculty of Veterinary Medicine;

${ }^{3}$ Health Sciences Library, University of Calgary, Calgary, Alberta

Correspondence: Dr CS Ribble, Centre for Coastal Health, 900 Fifth Street, Nanaimo, British Columbia V9R 5S5. Telephone 250-753-3245, fax250-740-6256,e-mail cribble@ucalgary.ca

Reprints: National Collaborating Centre for Infectious Diseases, 413-445 Ellice Avenue, Winnipeg, Manitoba R3B 3P5. Telephone 204-943-0051, fax 204-946-0927, e-mail nccid@icid.com 
variables to classify which incoming animals are at high risk of developing disease early during the feeding process to better target metaphylactic antimicrobial use immediately on arrival. Unfortunately, we did not notice much else in the literature in terms of managing high-risk animals at arrival, other than to avoid purchasing them - an approach that was only useful for owners of relatively small feedlots. There was much less published on other variables such as pen hygiene, pen sizes or densities, or movement of sick animals within the feedlot, which could be used to help feedlot managers improve their present methods of disease control.

A significant body of opinion advocated for a comprehensive and multimethod approach to infection control, but clinical trials or systematic evaluation of the effectiveness of infection control practices were limited almost exclusively to evaluating the effects of vaccinating animals before or at the time of arrival to the feedlot, and evaluating the effects of treating animals using different antimicrobial regimens on arrival (metaphylaxis) or after arrival on morbidity, mortality and growth. Most articles on AMR itself were microbial ecological studies of enteric organisms of public health concern as opposed to cattle pathogens of high concern. These articles dealt to a large degree with Escherichia coli, and to a lesser extent with Salmonella and Campylobacter species. Most were cross-sectional in nature and, thus, did not provide controlled evaluation of management methods to prevent or reduce AMR. Several of the observational risk factor studies found that the risk of pneumonia-related morbidity and mortality in calves increased significantly in the fall, when the sale of freshly weaned calves reached its peak. Furthermore, some found that this risk increased significantly as the fall progressed - a phenomenon dubbed the November effect' by Canadian researchers in the early 1990s. This finding coincided with the observations of some feedlot owners that the effectiveness of their metaphylactic antimicrobial strategies for high-risk calves seemed to decrease as the fall progressed. Studies are needed to explore how real and widespread this so-called 'November effect' might be across the industry, how much AMR might have to do with the phenomenon, and ultimately, what to do about it. These studies should include comparisons of how different animal management strategies within the feedlot (in terms of pen hygiene, pen densities, nutrition and animal movement, as well as different antimicrobial use strategies) could effectively decrease the 'November effect.'

Vaccination on arrival for pneumonia seemed to have some effect, although it appeared to be less than that for the concurrent strategy of antimicrobial metaphylaxis. This is not surprising given that many calves arrived at the feedlot already harbouring disease before vaccination. However, the true effectiveness of vaccination on arrival has been hampered by design faults present in many articles. Furthermore, given that many vaccine field trials were performed in which all high-risk animals received metaphylactic antimicrobials on arrival, it is difficult to know how well the vaccines would work in the absence of antimicrobial metaphylaxis. There was also some evidence that several weeks before being shipped to a feedlot, vaccination of calves at their source cow-calf farms reduced disease at the feedlot, but the consistency and size of the effect was difficult to establish, at least in part, due to design challenges. This, combined with a historical inability of the auction market transfer system to consistently provide cowcalf operators with a premium to vaccinate their calves means that prevaccination and preconditioning have not become effective disease management strategies for feedlot owners in North America.

Liver abscesses are one of the most commonly cited reasons for the use of in-feed antimicrobials in feedlots. Only 11 articles specifically dealt with liver abscesses. Attempts at vaccination for this bacterial disease have had, at best, mixed results. In the absence of effective vaccination, and given current feeding protocols that require rapid transition to high-energy feeds, medicated feeds remain the most common approach to reducing the prevalence and severity of liver abscesses. There is a lack of studies systematically examining the effect of feeding antimicrobials on AMR under commercial conditions.

We conclude that, with the possible exception of vaccination against some pathogens on or before arrival, there were no intervention studies published in the past 20 years that provided convincing evidence of useful management practices for large modern feedlots that reduced the incidence of illness and mortality from bacterial pneumonia, and did not also involve the administration of antimicrobials. Observational studies provided useful information as to what constituted a high-risk animal on arrival to the feedlot, and this information has been used to target antimicrobial metaphylaxis toward the higher risk groups. Future observational and intervention studies designed to explore the effectiveness of disease management practices alternative to antimicrobial use should be encouraged. The long-term effects of metaphylactic antimicrobial use in the feedlot on treatment efficacy and AMR should be examined within and across different feedlots. Finally, contact with researchers already exploring antimicrobial cycling or rotation in human hospital settings should be encouraged because this may provide insights or solutions that have not already been attempted on a commercial feedlot. Design challenges in both settings could be examined to explore how cross-fertilization of ideas could help research to progress in both settings. 


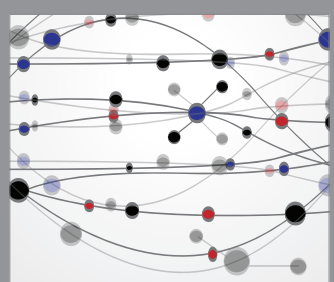

The Scientific World Journal
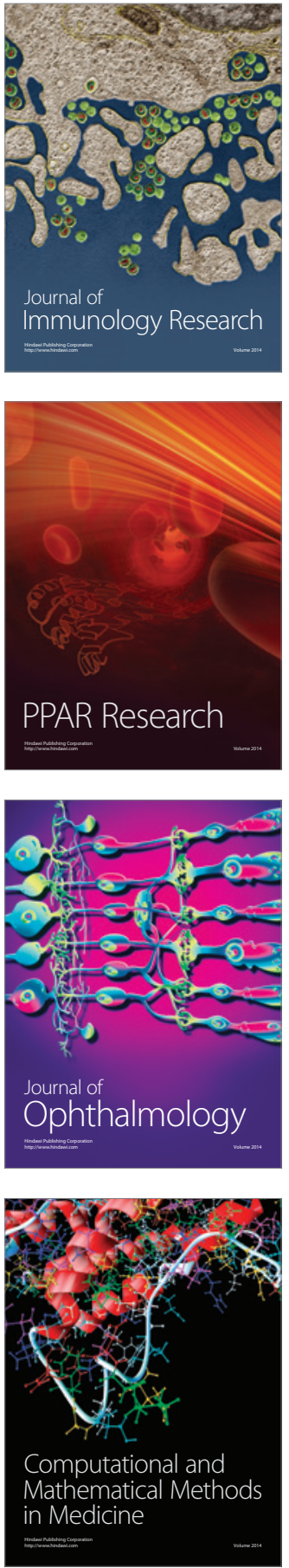

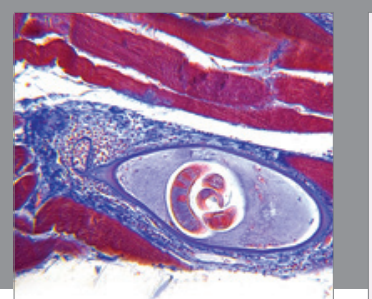

Gastroenterology Research and Practice

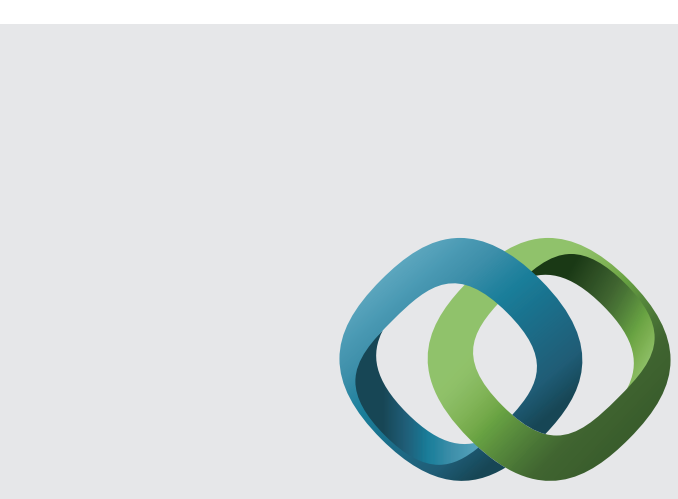

\section{Hindawi}

Submit your manuscripts at

http://www.hindawi.com
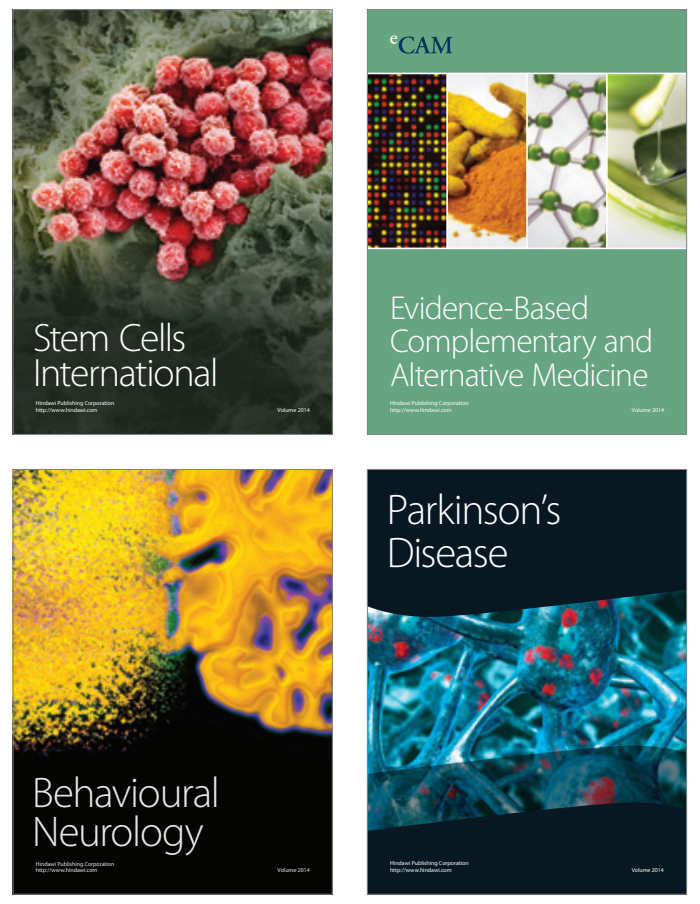
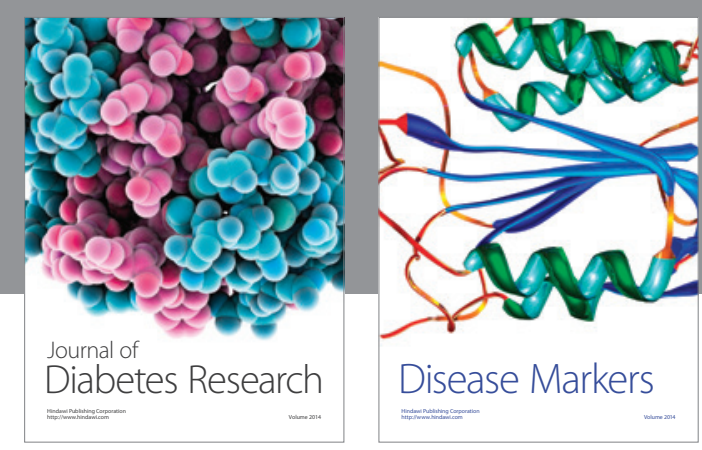

Disease Markers
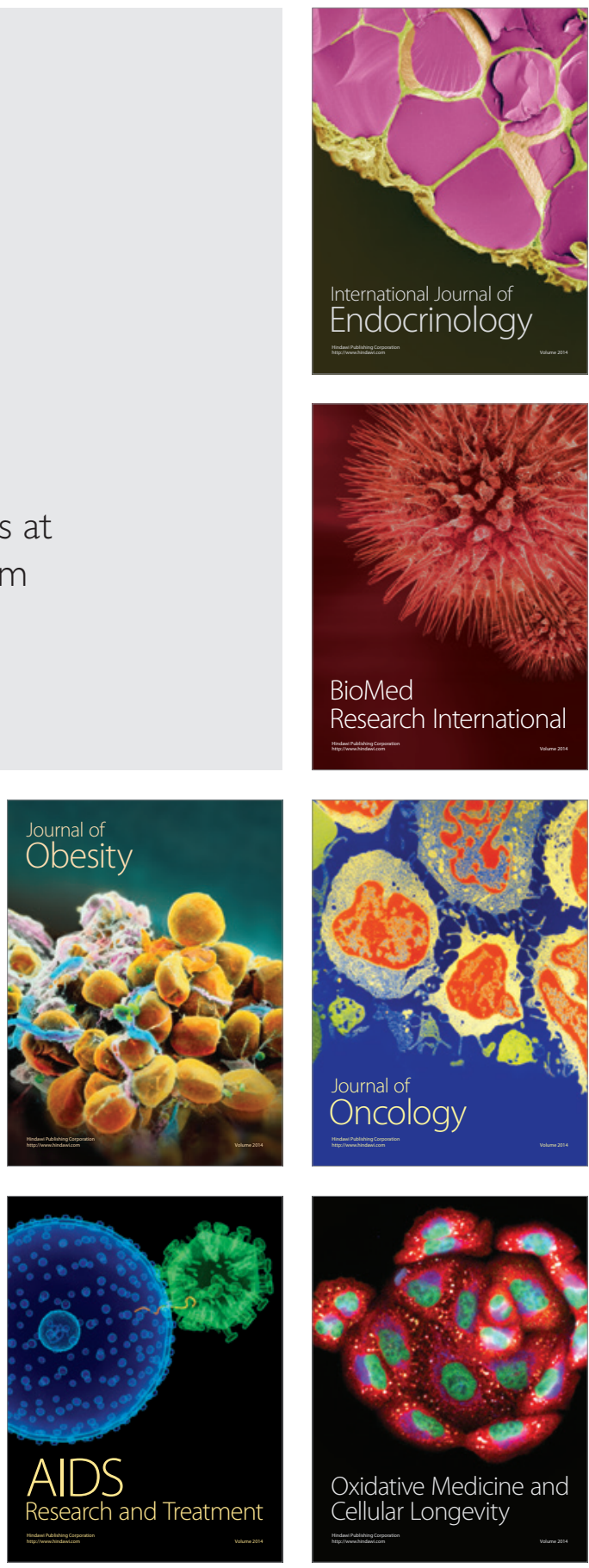